\title{
El Estrés Hídrico en Cítricos (Citrus spp.): Una revisión
}

\author{
Water stress on citric fruit (Citrus spp.): a review
}

O estresse hídrico em cítricos (Citrus spp): Uma revisão

\author{
Javier E Vélez ${ }^{1}$, Javier G Álvarez-Herrera ${ }^{2}$ Oscar H Alvarado-Sanabria ${ }^{3}$
}

1 Ing Agrícola, MSc, PhD, Departamento de Ingeniería Civil y Agrícola. Universidad Nacional de Colombia, Bogotá.

2 Ing Agrícola, MSc, Universidad Pedagógica y Tecnológica de Colombia, Tunja. Grupo de Investigaciones Agrícolas

3 Ing Agrónomo, Universidad Pedagógica y Tecnológica de Colombia, Tunja. Grupo de Investigaciones Agrícolas.

Email: jgalvarezh@gmail.com

Recibido: enero 02 de 2012.

Aceptado: mayo 25 de 2012

\section{Resumen}

Los cítricos constituyen el principal producto frutícola a nivel mundial por sus ventajas y beneficios. Una humedad adecuada y homogénea en el suelo garantiza su calidad y producción, incluso en los países subtropicales donde existen distribuciones uniformes de la precipitación. La mayoría de las áreas de cítricos cuentan con infraestructura de riego localizado, lo que incrementa la eficiencia en el uso del agua. Sin embargo, la presión sobre los recursos hídricos hace necesario seguir estudiando el comportamiento ante condiciones adversas para manejar eficientemente la programación del riego. En este artículo se presenta el efecto del déficit hídrico en los cítricos. Además de algunas respuestas fisiológicas y anatómicas que manifiestan los cítricos a este déficit y que efecto tiene un estrés hídrico sobre la producción y el crecimiento vegetativo. Por último se recopilan algunos resultados de la aplicación de riego deficitario como una forma de ahorrar agua sin afectar la producción.

Palabras clave: agua, evapotranspiración, árboles frutales, riego deficitario, estrés hídrico.

\begin{abstract}
Citric fruit represent the main fruit product worldwide due to their advantages and benefits. Adequate and homogenous moisture in the soil ensures consistent fruit quality and production, even in subtropical countries having patterns. Most citric-growing areas have drip irrigation infrastructure, thereby increasing water-use efficiency. However, pressure on water resources means that water use in adverse conditions must continue to be studied to ensure efficient irrigation scheduling. This paper deals with the effect of water stress on citric plants, as well as some of their physiological and anatomical responses to such deficit and what effect hydric stress has on vegetable production and growth. Some results are compiled regarding deficit irrigation (DI) as a way to save water without affecting production.
\end{abstract}

Key words: water, evapotranspiration, fruit tree, deficit irrigation, water stress.

\section{Resumo}

Os cítricos constituem o principal produto frutícola no mundo pelas suas vantagens e benefícios. Uma umidade adequada e homogênea no solo garante sua qualidade e produção, ainda nos países subtropicais onde existe distribuição uniforme da precipitação. A maioria das áreas citrícolas, contam com infraestrutura de risco localizado, o que incrementa a eficiência 
no uso da agua. Porém, a pressão sobre os recursos hídricos faz necessário estudar o comportamento em condições adversas para manejar eficientemente o planejamento da irrigação. Nesta revisão apresenta-se o efeito do déficit hídrico nos cítricos. Além disto, algumas respostas fisiológicas e anatômicas pelos cítricos ao déficit e o efeito do estresse hídrico sobre a produção e o crescimento vegetativo. Finalmente, apresentam-se alguns resultados da aplicação de irrigação deficitária, como forma de poupar água sem afetar a produção.

Palavras chave: agua, evapotranspiração, arvores frutais, irrigação deficitária, estresse hídrico.

\section{Antecedentes}

Los cítricos constituyen el principal producto frutícola, alcanzando una producción de 123.694.474 Tm y ocupando un área de 8.645.339 para el año 2010 (FAOSTAT, 2010). La producción y el consumo de jugo de cítricos han influido en el crecimiento sostenido del sector en los últimos decenios. Los principales países productores de estas frutas son china, Brasil, India y Estados Unidos con el 53\% de la producción mundial. Con el $56 \%$ de la producción, la naranja es el fruto más producido en relación a los demás cítricos (FAOSTAT, 2010). Esta fruta se consume en fresco y en forma procesada (principalmente como jugo). Su consumo en forma de jugo ha aumentado en los últimos años reemplazando el consumo de la fruta fresca, debido principalmente a la calidad del sabor y a avances tecnológicos en transporte y almacenamiento (FAO, 2001).

Los cítricos son árboles de hoja perenne, poseen una marcada competencia entre el crecimiento vegetativo y productivo, un sistema radicular relativamente superficial y un gran desarrollo del área foliar. Se adaptan a diversas situaciones ecológicas comprendidas entre el Ecuador y latitudes ligeramente superiores a los $40^{\circ}$ $\mathrm{N}$ y S. Estos árboles necesitan suelos permeables, humedad tanto en el suelo como en la atmósfera y unas temperaturas cálidas, alcanzan su máximo desarrollo en las áreas subtropicales (30-40 latitud $\mathrm{N}$ y S). En estas áreas la producción es estacional y la calidad del fruto para el consumo en fresco es excelente, ya que tiene una coloración anaranjada o amarilla muy atractiva y aunque el contenido de jugo no es muy alto éste tiene una relación de azucares y ácidos muy agradable al paladar. En las regiones tropicales desde el Ecuador hasta $24^{\circ}$ latitud $\mathrm{N}$ y S la calidad del fruto es muy variable y depende de los microclimas y la altitud. La producción es casi continua a lo largo del año y generalmente los frutos no alcanzan su color característico. Estos son jugosos, muy dulces, poco ácidos y se destinan principalmente al consumo local (Agustí, 2003).

\section{El riego en los cítricos}

Cuando el agua aportada por la lluvia es inferior a la evapotranspiración potencial del cultivo es necesario un suministro adicional por medio del riego para obtener cosechas rentables. El abonado, la poda y las labores culturales favorecen la producción lo que aumenta el volumen, calidad o regularidad de la misma, pero ninguna de las operaciones indicadas es absolutamente indispensable como el riego, por lo que es la operación cultural de mayor importancia en los huertos de cítricos. Está comprobado que mantener una humedad adecuada en el suelo durante el ciclo del cultivo garantiza una producción alta (Shalhevet y Levy, 1990) y una mejora en la calidad, incluso en los países subtropicales donde existen distribuciones uniformes de la precipitación.

Shalhevet y Levy (1990) recomiendan aplicaciones de

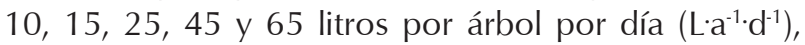
desde el primero al sexto año respectivamente. Desde el sexto en adelante se recomienda $100 \mathrm{~L}^{-1} \mathrm{a}^{-1} \cdot \mathrm{d}^{-1}$. Bielorai (1982) obtuvo producciones de 100 tha $^{-1}$ de pomelo (Citrus grandis) Marsh Seedless en el desierto del Negev ubicado en Israel al aplicar $803 \mathrm{~mm}$ con riego localizado cada 3 días. Sánchez-Blanco et al. (1989) obtuvieron producciones de 57 tha $^{-1}$ en limoneros Verna (Citrus limonium) en Murcia (España) aplicando $625 \mathrm{~mm}$ a diario. Castel y Buj (1990) en naranja (Citrus sinensis) Salustiana en Valencia (España), aplicando $553 \mathrm{~mm}$ de agua diarios consiguieron producciones de 75 tha $^{-1}$.

En condiciones tropicales, Orduz-Rodríguez y Fisher (2007) reportaron que en mandarina "arrayana" (Citrus reticulata Blanco) injertada sobre mandarina Cleopatra de 6 años de edad en el departamento del meta (Colombia) los requerimientos hídricos anuales son de 1046 mm, los cuales se distribuyen uniformemente a lo largo del año a excepción de los meses de diciembre y febrero, en los cuales se presenta un déficit hídrico de $247 \mathrm{~mm}$. La falta de agua durante estos tres meses induce la posterior floración en el mes de marzo, por lo que en estas condiciones es necesario un déficit hídrico para inducir la formación de flores.

También en el departamento del Meta pero en un cultivo de 12 años de naranja Valencia injertado sobre mandarina Cleopatra, Garzón (2012) comprobó que al regar con el $100 \%$ y el $50 \%$ de la evapotranspiración potencial la producción fue de 27 tha-1.y 22 tha $^{-1}$ res- 
pectivamente, en relación a 20 tha $^{-1}$ del testigo. Esta poca diferencia entre tratamientos se debe a que en este departamento solo hubo déficit hídrico en los meses de enero y febrero, lo que provocó que el testigo recuperara su potencial hídrico y la sequía no afectara la producción.

\section{Respuesta de los cítricos al estrés hídrico}

El agua es extraída a través del sistema radicular y transportada por la corriente xilemática, luego se distribuye como parte constituyente de la planta hasta alcanzar la atmósfera mediante el proceso de transpiración, que se da en respuesta al gradiente de energía aportada por la radiación solar (Salisbury y Ross, 2000). El crecimiento y desarrollo de la planta está influenciado por el medio ambiente, especialmente por la disponibilidad de agua, debido a que esta cumple un papel vital, pues interviene prácticamente en todos los procesos fisiológicos.

Un déficit hídrico afecta negativamente las funciones fisiológicas como: fotosíntesis, respiración, reacciones metabólicas y anatómicas, crecimiento, reproducción, desarrollo de semillas, absorción de nutrientes minerales, transporte de asimilados y producción (Quiñones et al., 2007; García-Sánchez et al., 2003; Ortuño et al., 2004). El efecto dependerá de la intensidad de la sequía, de la duración de la misma y de la época en que ocurre dentro del ciclo del cultivo, esto ocurre tanto en cítricos (Ballester et al., 2011) como en otras especies (Vaux y Pruitt, 1983; Reichstein et al., 2002)

Los cítricos son árboles de hoja perenne que transpiran durante todo el año, tienen un periodo de crecimiento efectivo que alterna con otro de inactividad vegetativa. El crecimiento y producción dependen del balance entre la demanda evaporativa y el suministro de agua, así como de una adecuada nutrición. Estas condiciones están ligadas al ambiente, el cual favorece o perjudica la expresión de genes deseables en las plantas cultivadas (Sinclair, 1984).

Las plantas constantemente están sometidas a condiciones ambientales desfavorables por lo que desarrollan mecanismos de protección. La adaptación al estrés hídrico puede ser de varios tipos dependiendo de los niveles de dicho estrés. Estas adaptaciones combinan factores anatómicos y fisiológicos que limitan el almacenamiento y transporte del agua por la planta. Como consecuencia del estrés hídrico se producen una serie de respuestas en cítricos como: disminución en la conductancia de los estomas, con lo cual se evita la perdida de agua por transpiración, abscisión foliar (Pérez-Pérez et al., 2008), disminuyendo el área foliar y por tanto la superficie de transpiración y adaptaciones a nivel metabólico y osmoregulación, en las células acumulan una serie de solutos compatibles con el funcionamiento normal del mecanismo celular (Hockema y Etxeberria, 2001; Barry et al., 2004; Navarro et al., 2010).

Las hojas están adaptadas para conservar el agua gracias a sus distintas capas cerosas. Las hojas jóvenes poseen un reducido control sobre la transpiración estomática y son más pobres en ceras epicuticulares y cutina que las hojas viejas, esta abundante cutícula cerosa en ocasiones ocluye los estomas, lo que representa una reducida pérdida de agua (Agustí, 2003). Los estomas están generalmente en el envés y en condiciones de estrés hídrico severo se cierran, restringiendo el intercambio de $\mathrm{CO}_{2}$ y la pérdida de agua (Ortuño et al., 2004; Tudela y Primo-Millo, 1992); Durante la deshidratación también se producen cambios en los ángulos de inclinación de las hojas. Pero el enrollamiento foliar es probablemente la manifestación más evidente del déficit hídrico, debido a que reduce la superficie foliar y la radiación interceptada, disminuyendo la transpiración.

La eficiencia en la utilización del agua por parte de las plantas se define como la relación entre las unidades de materia seca total producida por unidad de agua consumida, o en términos de fotosíntesis como miligramos de dióxido de carbono $\left(\mathrm{CO}_{2}\right)$ absorbidos por gramos de agua empleados en el proceso de transpiración (Bancon, 2004). Esta eficiencia además de depender de factores climáticos también se ve afectada por el material vegetal usado y la fenología del mismo. En el cultivar 'Lane late' de naranja Pérez-Pérez et al. (2010) encontraron que el porta injertos "Carrizo" es más eficiente en la relación producción agua ${ }^{-1}$ suministrada que "Cleopatra". Por otra parte, en mandarina "Clementina de Nules", al someterla a estrés hídrico durante dos años consecutivos, la eficiencia no aumento significativamente en el periodo fenológico III del fruto (Pérez-Pérez et al., 2009), revelando que en esta fase fenológica no es significativo el ahorro de agua usando riego deficitario.

Como respuesta al estrés hídrico se detiene el desarrollo de la planta, hay marchitamiento de las hojas, reducción del potencial hídrico, de la conductancia estomática, de la asimilación de $\mathrm{CO}_{2}$ y de la conductividad hidráulica de la raíz (Tudela y Primo-Millo, 1992; Pérez-Pérez et al., 2009). Cuando la pérdida de agua es rápida debido a altas temperaturas y vientos fuertes y secos se puede producir estrés hídrico en la planta aún con humedad aceptable en el suelo. Este estrés afecta de diferente manera e intensidad los distintos 
estados de crecimiento. Un ejemplo es que afecta la inflorescencia y la fecundación, produciendo un aborto floral y abscisión del fruto (González-Altozano y Castel, 2003).

Savé et al. (1995) en naranjo y tangor encontraron que ante condiciones de estrés hídrico severo se desarroIlan mecanismos de ajuste elástico. Este mecanismo consiste en el aumento de la elasticidad de las paredes celulares con el objeto de mantener la turgencia celular, lo que facilita al igual que en el ajuste osmótico la continuidad de procesos esenciales como la utilización de nutrientes y asimilados (Huang et al., 2000), la fotosíntesis y por lo tanto el crecimiento en general. Cuando el déficit hídrico es moderado no se desarroIlan mecanismos de ajuste elástico (Savé et al., 1995).

El déficit hídrico es un gran perturbador del balance hormonal de muchas especies vegetales alterando significativamente los procesos fisiológicos del crecimiento y desarrollo. En cítricos, Montoliu (2010) afirma que el estrés hídrico aumenta el contenido de ácido abscísico, ácido jasmónico y ácido salicílico, mientras que el contenido de giberelinas disminuye rápidamente en las hojas sometidas a estrés hídrico, aunque se recobra nuevamente al reanudar el riego (Mahouachi et al., 2005). Desde el punto de vista de producción de etileno los mayores efectos del estrés hídrico se manifiestan en la senescencia foliar, la degradación de la clorofila y la abscisión de hojas y frutos.

El ácido abscísico (ABA) tiene una doble función en la protección de los cítricos frente al estrés hídrico: a corto plazo reduce la transpiración debido a que el suministro de ABA a las hojas produce el cierre inmediato de los estomas (Mahouachi et al., 2005), mientras que a largo plazo induce la síntesis de proteínas que aumentan la tolerancia de la planta a la desecación. En condiciones de estrés hídrico la biosíntesis de ABA se induce por la pérdida de la turgencia. Sin embargo, los estomas a menudo se cierran antes de que se produzca el aumento del ABA en la hoja. El estrés reduce el gradiente de potencial de Hidrogeno $(\mathrm{pH})$ entre el citoplasma y el apoplasto y debido a que el contenido de $\mathrm{ABA}$ es mayor donde el $\mathrm{pH}$ es más elevado se promueve el flujo de $A B A$ desde la célula. Por tanto, los niveles de $A B A$ aumentan en el apoplasto antes que en cualquier otro lugar de la hoja. Las evidencias indican que el ABA producido en las raíces puede actuar como una señal mediante la cual las plantas regulan su estado hídrico en los suelos secos (Mahouachi et al., 2005; Zhang y Outlaw, 2001). La función principal del ABA sintetizado en respuesta a la sequía parece ser la regulación de la tolerancia a éste estrés mediante la expresión de genes que confieren protección frente a la desecación (Gimeno et al., 2009).

\section{Efectos del estrés sobre el desarrollo vegetativo en cítricos}

Los meristemos hacen posible el crecimiento primario por medio de la división celular o adición de nuevas células y están estrechamente relacionados con las hormonas que son promotoras de esta división. El crecimiento secundario de órganos vegetativos tiene su origen en el cambium vascular, donde se diferencian en células del floema y del xilema. La expansión celular se debe al potencial de turgencia, suministro de sales minerales, agua y materia elaborada (Taiz y Zeiger, 2002), debido a esto, el crecimiento es uno de los procesos más afectados por el déficit hídrico.

El desarrollo vegetativo en varios patrones de cítricos es muy sensible al déficit hídrico (Rodríguez-Gamir et al., 2010). La disminución de la superficie foliar como consecuencia de un menor crecimiento es un mecanismo adoptado por la planta para reducir la pérdida de agua por transpiración tanto en mandarino (Tudela y Primo-Millo, 1992) como en otros árboles (Potters et al., 2007). Cítricos regados por debajo de sus requerimientos hídricos no alcanzan el tamaño de las plantas que si reciben un suministro adecuado (GarcíaOrellana et al., 2007). Como consecuencia del déficit hídrico se redujo el tamaño foliar en pomelos (Levy et al., 1978) y en Clementina de Nules (Ginestar y Castel, 1996b). Las hojas jóvenes tienen potenciales osmóticos mayores que las hojas viejas y consecuentemente pierden la turgencia antes que las hojas maduras. Así pues, el crecimiento de hojas nuevas es muy sensible al déficit hídrico (Syvertsen, 1985). La emisión de nuevas hojas desaparece por completo durante periodos prolongados sin riego. Pero al reanudarlo, rápidamente se inicia una intensa brotación y el crecimiento vegetativo se recupera (González-Altozano y Castel, 2003). La senescencia, abscisión y caída de las hojas son medios drásticos para limitar la transpiración en caso de sequía severa. Sin embargo, ésta adaptación conlleva un retraso importante en el crecimiento y desarrollo de la planta como lo comprobaron Gómez-Cadenas et al. (1996) en mandarina "Clementina de Nules".

El déficit hídrico no sólo reduce la actividad meristemática y el alargamiento radicular, sino que aumenta la suberización de las raíces con el consiguiente efecto negativo sobre la absorción de agua y nutrientes minerales. La capacidad de la planta para satisfacer plenamente la demanda evaporativa de la atmósfera depende del volumen de suelo explorado por las raíces, distribución y densidad del sistema radicular, 
la resistencia al flujo de agua en el sistema radical, la resistencia al movimiento en el sistema aéreo, el contenido y distribución de humedad en el suelo y las propiedades hidráulicas del suelo. La profundidad radicular en suelos profundos y bien drenados está entre 0.6 a $1.5 \mathrm{~m}$ y la mayor extracción de agua y nutrientes ocurre en los primeros $60 \mathrm{~cm}$ (Shalhevet y Levy, 1990; Sheng et al., 2002; Quiñones et al., 2007). Se ha comprobado en árboles de distintas edades que una baja disponibilidad de agua en el suelo reduce el crecimiento radicular (Rodríguez-Gamir et al., 2010; Melgar et al., 2010). Mitchell et al. (1986) encontraron que los mayores rendimientos en riego deficitario controlado (RDC) se presentaron en los cultivos de mayor densidad de plantación, sugiriendo que el RDC es más eficiente cuando el crecimiento radicular está limitado.

La iniciación, así como la diferenciación de las estructuras vegetativas y reproductivas y el alargamiento celular una vez diferenciadas las células son muy sensibles al estrés hídrico y al igual que los procesos fisiológicos no son afectados necesariamente con la misma intensidad (Gonzales-Altozano y Castel, 2003).

En Clementina de Nules, en condiciones mediterráneas existen de cuatro a cinco crecimientos o brotaciones al año. El primero ocurre en primavera y en él se da la floración que produce la fruta de temporada. Durante el verano ocurren dos o más crecimientos vegetativos y a finales de otoño el cuarto, que normalmente es vegetativo. Con bajas humedades volumétricas en los tratamientos de estrés moderado el crecimiento se reduce debido a la disminución del agua aplicada (Levy et al., 1978; Ballester et al., 2011).

Higelman (1977) encontró en naranjos Valencia que el efecto de estrés hídrico se manifiesta de forma clara en el crecimiento vegetativo. Se puede limitar el desarrollo de la copa controlando el grado del déficit hídrico (Wiegand y Swanson, 1982). De igual forma, se pueden inducir brotaciones fuera de estación aplicando riego después de un déficit hídrico (GonzálezAltozano y Castel, 2000). La falta de agua da menos vigor a los árboles, y la defoliación y desecación apical se relacionan con la aportación de agua directa o indirectamente (Del Rivero, 1994). Uno de los objetivos del RDC en algunos cultivos es evitar que el exceso de vigor afecte la fructificación. Reduciendo el riego durante la brotación y el desarrollo de las ramas se limita el crecimiento vegetativo, favoreciendo el abastecimiento de la planta durante el crecimiento del fruto.

El crecimiento del tronco se reduce de forma proporcional a la reducción del suministro hídrico (Vélez et al., 2007). Existen estudios que comprueban que el estrés hídrico reduce el crecimiento del tronco en árboles jóvenes de limón (Ortuño et al., 2004) y otras especies (Levy et al., 1978). Reducciones en el crecimiento del tronco por riegos deficitarios han sido reportadas por Bielorai et al. (1981) en naranja, Bielorai (1982) en pomelo, y Castel (1994) en mandarina. En melocotonero, cambios estacionales inducen importantes variaciones sobre los valores de la máxima contracción del tronco (MCD) (Marsal et al., 2002), contrario a lo encontrado por Ginestar y Castel (1996) en Clementina de Nules regados por goteo, donde solo en el periodo final del verano observaron que el tratamiento que aportaba menos agua tuvo oscilaciones diurnas mayores a la del control.

\section{Efectos del estrés en la producción y calidad del fruto}

En los cítricos en condiciones de clima mediterráneo la falta de agua constituye un factor limitante del crecimiento del fruto (Pérez-Pérez et al., 2009) y en general de la producción (Ginestar and Castel, 1996b; Romero et al., 2006). Maotani et al. (1977) encontraron en mandarinas Satsuma que el crecimiento del fruto y del tronco era más sensible al estrés que el de las hojas. Así, con valores de potencial de agua al amanecer (Чa) de -0.8 MPa, el crecimiento del fruto cesaba, mientras que el de la hoja continuaba hasta valores de -1.5 a -2.0 MPa. Un periodo crítico es la fase inicial de crecimiento del fruto, donde aumenta la caída y provoca la disminución de la tasa de crecimiento, determinando el tamaño final del mismo (Ginestar y Castel, 1996b). La caída de frutos en ciertas épocas de año está fomentada entre otras cosas por una situación de déficit hídrico (Agustí, 2003). En naranjo "Salustiana", Castel y Buj (1990) encontraron que el déficit hídrico durante la fase de floración y cuajado del fruto ocasiona el aumento de caída de flores y frutos pequeños, condicionando el número de frutos y disminuyendo los volúmenes de producción. Con aportes del 90\% y $60 \%$ de las necesidades de riego no encontraron diferencias en el número de frutos por árbol, aunque, la producción disminuyó de un $5 \%$ a un $15 \%$, respectivamente. Los frutos de estos tratamientos mostraron niveles de acidez y sólidos solubles mayores.

En condiciones tropicales con un régimen de lluvias monomodal, Garzón (2012) afirma que el uso de riego luego de una época seca de dos meses mejora la calidad del fruto aumentando su diámetro ecuatorial y el número de frutos por árbol. Sin embargo, otras características como el índice de madurez y cantidad de jugo no se ven afectadas. Esto demuestra que aun 
en condiciones homogéneas de precipitación el uso de riego da un valor agregado a la producción.

El control del estado hídrico del suelo induce en los árboles una precocidad en la maduración de los frutos. Los aportes continuos de agua favorecen la homogeneidad de los frutos frente a la heterogeneidad observada en árboles regados con mayores dosis y menor frecuencia (Gómez Aparisi, 1991). Un déficit hídrico moderado durante el llenado y maduración puede ser conveniente para realizar cambios internos en la calidad de la fruta, principalmente aumentando el contenido de sólidos solubles y ácidos (Peng y Rave, 1998).

El déficit de agua en verano se utiliza en algunas variedades de especies de cítricos como limonero y lima para inducir la floración fuera de temporada. PerezPerez et al. (2009) en naranja "lane late" mediante la reducción del aporte hídrico presentaron un retraso en la cosecha sin afectar la producción.

Ginestar y Castel (1996b) determinaron que los periodos fenológicos más sensibles a la reducción de los aportes de agua en Clementina de Nules fueron la floración y la fructificación, lo que afectó significativamente la producción y la calidad del fruto.

El riego deficitario controlado (RDC) permite un ahorro de agua que se traduce en menor consumo de energía, disminución de los costos de producción, aumento de la rentabilidad, mayor eficiencia en la utilización del recurso hídrico y disminución de riesgos de contaminación por percolación profunda (Vélez, 2011).

\section{Referencias}

Agustí M. 2003. Citricultura. Segunda edición. Ed. Mundiprensa (España). 423p.

Arnon I. 1979. Optimizing yields and water use in Mediterranean agriculture. Soils in Mediterranean type climates and their yield potential. Int. Potash Institute.

Ballester C, Castel J, Intrigliolo DS, Castel JR. Response of Clementina de Nules citrus trees to summer deficit irrigation. Yield components and fruit composition. Agricultural Water Management, 2011; 98: 1027-1032.

Bancon M. 2004. Water use efficiency in plant biology. First edition. Blackwell Publishing Ltd. (EUA). 344p.

Barry GH, Castle WS, Davies FS. Rootstocks and plant water relations affect sugar accumulation of citrus fruit via osmotic adjustment. Journal of the American Society for Horticultural Science, 2004; 129: 881-889.

Bielorai H, Dasberg S, Erner Y, Brum M.. The effect of various soil moisture regimes and fertilizer levels on citrus yield under partial wetting of the root zone. Proc. Int. Soc. Citriculture Cong, (Tokyo) 1981;1: 585-589.
Bielorai $\mathrm{H}$. The effect of partial wetting of the root zone on yield and water use efficiency in a drip-and sprinkler-irrigated mature grapefruit grove. Irrigation Science, 1982; 3: 89-100.

Castel JR, Buj A. Response of Salustiana oranges to high frequency deficit irrigation. Irrigation Science, 1990; 11: 121-127.

Castel JR. Response of young clementine citrus trees to drip irrigation. I. Irrigation amount and number of driper. The Journal of Horticultural Science, 1994; 69 (3): 481-489.

Del Rivero JM. 1994. Efecto de factores naturales y de origen mixto sobre los cítricos, $2^{\underline{a}}$ edición ampliada. Edit. Universidad Politécnica de Valencia. 328p.

FAO. Food and Agriculture Organization of the United Nations. 1988. Zumos cítricos. Tendencias y perspectivas de la producción mundial y del mercado internacional. Estudio FAO, desarrollo económico y social No. 78. Roma.

FAO. Food and Agriculture Organization of the United Nations. 2006. Informe estadístico de la producción agrícola en el mundo. Roma.

FAO. Food and Agriculture Organization of the United Nations. Thomas H. Spreen. 2001. Proyecciones de la producción y consumo mundial de los cítricos para el 2010. Florida, Estados Unidos.

FAOSTAT. 2010. Producción mundial de cítricos año 2010. [Consultado el 26 de Mayo de 2012] URL: http://faostat.fao.org/ site/567/DesktopDefault.aspx?PageID=567\#ancor.

García-Orellana Y, Ruiz-Sánchez MC, Alarcón JJ, Conejero W, Ortuño MF, Nicolás E, Torrecillas A. Preliminary assessment of the feasibility of using maximum daily trunk shrinkage for irrigation scheduling in lemon trees. Agricultural Water Management, 2007; 89: 167-171.

García-Sánchez F, Carvajal M, Porras I, Botía P, Martínez V. Effects of salinity and rate of irrigation on yield, fruit quality and mineral composition of 'Fino 49' lemon. Europ. J. Agronomy, 2003; 19: 427-437.

Garzón DL. 2012. Evaluación de la influencia del déficit hídrico en el crecimiento y desarrollo de la naranja "valencia" (Citrus sinensis O) en el piedemonte llanero de Colombia. Tesis de maestria. Universidad Nacional de Colombia (Bogota).

Gimeno J, Gadea J, Forment J, Pérez-Valle J, Santiago, J, MartínezGodoy MA, Yenush L, Bellés JM, Brumós, J, Colmenero-Flores, JM, Talón M, Serrano R. Shared and novel molecular responses of mandarin to drought. Plant Molecular Biology, 2009; 70 (4): 403-420.

Ginestar C, Castel JR. Response of young Clementine citrus trees to water stress during different phenological periods. The Journal of Horticultural Science, 1996a; 71 (4): 551-559.

Ginestar C, Castel JR. Utilización de dendrómetros como indicador de estrés hídrico en mandarinos jóvenes regados por goteo. Riegos y Drenajes XXI, 1996b; 89: 40-46.

Gómez-Aparisi J. El riego en la producción frutal. Necesidades hídricas: Riego tradicional y microirrigación. Riegos y Drenajes XXI, 1991;48: 18-24. 
Gómez-Cadenas A, Tadeo FR, Talon M, Primo-Millo E. Leaf abscission induced by ethylene in water stressed intact seedling of Cleopatra mandarin requires previous abscisic acid accumulation in roots. Plant Physiol, 1996; 112: 401-408.

González-Altozano P, Castel JR. Regulated deficit irrigation in "Clementina de Nules" citrus trees. II: Vegetative effects. The Journal of Horticultural Science. Biotech, 2000;75 (4): 388-392.

González-Altozano P, Castel JR. Riego deficitario controlado en Clementina de Nules. II Efectos sobre el crecimiento vegetativo. Spanish Journal of Agricultural Research, 2003; 1(2): 93-101.

Hilgeman RH. Response of citrus to water stress in Arizona. Proc. Int. Soc. Citriculture Cong. (Orlando), 1977;1: 70-74.

Hockema, BR, Etxeberria E. Metabolic contributors to drought-enhanced accumulation of sugars and acids in oranges. Journal of the American Society for Horticultural Science. 2001; 126: 599-605.

Huang X, Huang $\mathrm{H}$, Gao F. The growth potential generated in citrus fruit under water stress and its relevant mechanisms. Scientia Horticulturae, 2000; 83: 227-24.

Levy $\mathrm{Y}$, Bielorai H, Shalhevet J. Long-term effects of different irrigation regimes on grape fruit tree development and yield. Journal of the American Society for Horticultural Science, 1978;103: 680-683.

Mahouachi J, Gómez-Cadenas, Primo-Millo E, Talon M. Antagonistic Changes between Abscisic Acid and Gibberellins in Citrus Fruits Subjected to a Series of Different Water Conditions. Journal of Plant Growth Regulation, 2005; 24:179-187.

Maotani T, Machida Y, Yamatsu K. Studies on leaf water stress in fruit trees. VI. Effects of leaf water potential on growth on Satsuma mandarin trees. Journal of the Japanese Society for Horticultural Science, 1977; 45: 329-334.

Marsal J, Gelly M, Mata MA, Rufat J, Girona J. Phenology and drought affects the relationship between daily trunk shrinkage and midday stem water potential of peach trees. The Journal of Horticultural Science. Biotech, 2002; 77: 411-417.

Martínez JA. 1999. El comportamiento del consumidor de cítricos: naranjas y mandarinas. Generalitat Valenciana. Conselleria $D^{\prime}$ Agricultura, Peixca I Alimentacio. Valencia. p. 7-265.

Melgar JC, Dunlop JM, Syvertsen JP. Growth and physiological responses of the citrus rootstock Swingle citrumelo seedlings to partial rootzone drying and deficit irrigation. Journal of Agricultural Science, 2010; 148 (5): 593-602.

Mitchell PD, Chalmers DJ, Jerie PH, Burge G. The use of initial withholding of irrigation and trees. Journal of the American Society for Horticultural Science, 1986; 111: 858-861.

Montoliu VA. 2010. Respuestas fisiológicas de los cítricos sometidas a condiciones de estrés biótico y abiótico aspectos comunes y específicos. Tesis Doctoral. Universitat Jaume I.

Naor A, Irrigation scheduling and evaluation of tree water status in deciduous orchards. Hortic. Rev, 2006; 32: 111-166.
Navarro JM, Pérez-Pérez JG, Romero P, Botía P. Analysis of the changes in quality in mandarin fruit, produced by deficit irrigation treatments. Food Chemistry, 2010; 119:1591-159.

Orduz-Rodríguez O, Fischer G. Balance hídrico e influencia del estrés hídrico en la inducción y desarrollo floral de la mandarina 'Arrayana' en el piedemonte llanero de Colombia. Agronomía Colombiana, 2007;25(2): 255-263.

Ortuño MF, Alarcón JJ, Nicolás E, Torrecilla A. Interpreting trunk diameter changes in young lemon trees under deficit irrigation. Plant Science, 2004; 167: 275-280.

Peng YH, Rave E. Effect of differing irrigation regimes on fruit quality, yield, fruit size and net $\mathrm{CO} 2$ assimilation of Mihowase Satsuma. The Journal of Horticultural Science. Biotech, 1998; 73: 229234.

Pérez-Pérez J, García JM, Robles P, Botía P. Economic analysis of navel orange cv. 'Lane late' grown on two different drought-tolerant rootstocks under deficit irrigation in South-eastern Spain. Agricultural Water Management, 2010; 97: 157-164.

Pérez-Pérez JG, Robles JM, Botía P. Influence of deficit irrigation in phase III of fruit growth on fruit quality in 'lane late' sweet orange. Agricultural Water Management, 2009; 96: 969-974.

Pérez-Pérez, J.G., Romero, P., Navarro, J.M., Botía, P. Response of sweet orange cr 'Lane late' to deficit irrigation in two rootstocks. I: Water relations, leaf gas exchange and vegetative growth. Irrigation Science, 2008; 26(5): 415-425.

Potters G, Pasternak TP, Guisez Y, Palme KJ, Jansen MAK. Stress-induced morphogenic responses: growing out of trouble? Trends in Plant Science, 2007;12(3): 99-105.

Quiñones A, Martínez-Alcántara B, Legaz F. Influence of irrigation system and fertilization management on seasonal distribution of $\mathrm{N}$ in the soil profile and on $\mathrm{N}$-uptake by citrus trees. Agriculture: Ecosystems and Environment, 2007;122: 399-409.

Reichstein M, Tenhunen JD, Roupsard O, Ourcival JM, Rambal S, Miglietta F, Peressotti A, Pecchiari M, Tirone G, Valentini R. Severe drought effects on ecosystem $\mathrm{CO} 2$ and $\mathrm{H} 2 \mathrm{O}$ fluxes at three Mediterranean evergreen sites: revision of current hypotheses. Global Change Biology. 8: 999-1017. Rev. Plant Physiol. Plant Mol. Biol. 2002; 49: 199-222.

Rodríguez-Gamir J, Primo-Millo E, Forner J.B, Forner-Giner MA. Citrus rootstock responses to water stress. Scientia Horticulturae. 2010;126(2): 95-102

Romero P, Navarro JM, Pérez-Pérez JG, García-Sánchez F, GómezGómez A, Porras I, Martínez V, Botía P. Deficit irrigation and rootstock: their effects on water relations, vegetative development, yield, fruit quality and mineral nutrition of Clemenules mandarin. Tree Physiology, 2006; 26: 1537-1548.

Salisbury F, Ross C. 2000. Fisiología de las Plantas. Vol 3. PARANINFO. 988p.

Sánchez-Blanco MJ, Torrecillas A, Del Amor F, León A. Effect of irrigation regimes on the stages of reproduction in Citrus limonum L. cv. Verna. Advances in Horticultural Science, 1989; 3:13-16. 
Savé R, Biel C, Domingo R, Ruiz-Sánchez MC, Torrecillas A. Some physiological and morphological characteristics of Citrus plants for drought resistance. Plant Science, 1995; 110:167-172.

Shalhevet J, Levy Y. 1990. Citrus trees. In: Stewart BA, Nielsen, DR. (eds.) Irrigation of Agricultural Crops. A.S.A. Madison, Wis. Mon. no 30. p. 951-986.

Sheng LY, Tomohisa Y, Mehmet A, Yoshinobu K, Shin-Ichi T. Short term effects of saline irrigation on evapotranspiration from lysimeter-grown citrus trees. Agricultural Water Management, 2002; 56:131-141.

Sinclair WH. 1984. The Biochemistry and Physiology of the Lemon and Other Citrus Fruits, Univ. Calf. Div. Agric. Nat. Res., Riverside, California. 946 p.

Syvertsen JP. Integration of water stress in fruit trees. Hort Science, 1985; 20 (6): 1039-1043.

Taiz L, Zeiger E. 2002. Plant Physiology. 3rd ed. Sinauer Associates Inc. Publishers, Sunderland, MA. 690p.

Tudela D, Primo-Millo E. 1-Aminocyclopropane-1-carboxylic acid transported from roots to shoots promotes leaf abscission in
Cleopatra mandarin (Citrus reshni Hort, ex Tan) Seedlings rehydrated after water stress. Plant Physiology, 1992; 100:131137.

Vaux JR, Pruitt WO. Crop water production functions. Advances in irrigation, 1983; 2:1-99.

Vélez-Sánchez, JE, Intrigliolo DS, Castel J. R. Programación de riego en base a sensores de medida del estado hídrico del suelo y de la planta. Revista UDCA Actualidad y Divulgación Científica, 2011; 14(2): 65-73.

Vélez JE, Intrigliolo DS, Castel JR. Scheduling deficit irrigation of citrus trees with maximum daily trunk shrinkage. Agricultural Water Management, 2007; 90: 197-204.

Wiegand CL, Swanson WA. Citrus response to irrigations. III. Tree trunk and canopy growth. J. Río Grande Valley Hortic. Soc, 1982; 35: 97-107.

Zhang SQ, Outlaw WH. Abscisic acid introduced into the transpiration stream accumulates in the guard cell apoplast and causes stomatal closure. Plant, Cell \& Environment, 2001; 24: 10451054. 